\section{Exotic woody plants inclining to escape in the Buda Arboretum under strong urban effect in Budapest}

\author{
Schmidt G.
}

Szent Istvan University Faculty of Horticultural Sciences, Department of Floriculture and Dendrology, H-1118Budapest,Villányi út35-43.E-mail:disz@omega.kee.hu

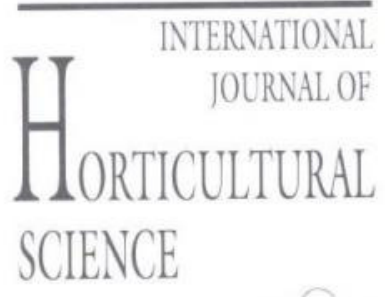

AGROINFORM

Publishing House, Hungary

Key words: arboretum, woody exotic species, ornamental trees and shrubs, introduction, escape, spreading

Summary: The Buda Arboretum of the Szent Istvan University is situated in the western-middle part of the city of Budapest. It contains 1640 taxa of woody plants on an area of 7.5 ha. The sheltered position and the urban micro- and meso-climate is favourable for warm-loving, even subtropical plants, of which many species not simply stay alive, but flower, bear fruits and are spreading by seedlings in suitable spots. These instances call our attention to the possibilities that urban climate offers for landscapers, but also to over 110 species, which can be garden escapes in the future. Full list of plants, which tend to escape in the Arboretum is given in the paper.

\section{Introduction and review of literature}

The Buda Arboretum of the Szent István University is situated in the western-middle part of Budapest at the southern slope of Gellért Hill between the streets Villányi út-Szüret u.-Somlói út. It belongs to the south-eastern part of the Buda Hills at the border of the Great Plain and the Transdanubian Hills. Annual precipitation is $600-620 \mathrm{~mm}$. the local climate is slightly arid. The majority of the plants thrive well only with irrigation. For sustaining water regime equilibrium, irrigation water equivalent of $150-200 \mathrm{~mm}$ precipitation is given during the vegetation period.

The soil forming rock is partly limestone and dolomite. but the most common are loamy, clayey chalky deposits. In this latter humus-carbonate and in some places brown forest soils have been developed. The original soil meanwhile has strongly eroded, the uppermost layers now are B or C. The present soil of the arboretum is therefore clayey, rich in $\mathrm{Ca}$ $(8-10 \%)$, poor in humus and alkaline (around $\mathrm{pH} 8.0$ ). Around the central building there are many bankings, the original profile cannot be separated.

The climate is much warmer than the country average, summers are hot, winters are mild, there are no frosts in May and in September. The vegetation period is therefore extended by 3-5 weeks in the autumn and in spring (Schmidt, 1993 and 1994; Hámori, 2000).

The territory of the Arboretum was once covered by vineyards, which were destroyed by the phylloxera-disease (root aphids) in the last century. First plantings were started during the winter of 1893-1894. That time about 1000 taxa of trees and shrubs were planted. Some of them are still alive, they are more than 100 years old (Schmidt, Keller \& Pintér 2000). Currently there are 1640 woody taxa on a 7.5 hectares area in the arboretum among which heat-loving and frostsensitive exotic species are one of the main attractions. Many of them tend to "escape" from culture: produce viable seed, which germinate and give rise to a secondary vegetation inside and sometimes also outside the borders of arboretum. Udvardy (1999a) studied the subspontaneous spreading of two interesting subtropical species, Diospyros lotus and Smilax excelsa in the arboretum.

The aim of the present paper is to study which (and which geobotanical groups) of exotic woody plants tend to breed by themselves and to escape in the strong urban environment of the Buda Arboretum created by the metropolis of Budapest.

\section{Material and method}

Several annotations have been made during the vegetation period for about 20 years. Seedlings of varieties and cultivars were identified upon the distance measured from the parent specimen and according to their frequency classified into one of the groups as follows:

$$
\begin{aligned}
& 1 \text { - } 1-2 \text { seedlings per year } \\
& 2 \text { - few seedlings } \\
& 3 \text { - many seedlings in some places } \\
& 4 \text { - many seedlings all over the Arboretum }
\end{aligned}
$$

The name of the plants are given according to the nomenclature of the Index Kewensis (Jackson et al., 1991). 


\section{Results}

The results of observations concerning spreading and escape of woody alien plants are shown in Table 1 .
According to the hardiness map of Krüssmann (1986-1986) Hungary is situated in winter-hardiness zones 6 and partially 7 . As seen on table 1., quite a lot of southern

Table 1 Spreading tendency of exotic trees and shrubs in the Buda Arboretum (legend of columns 2-3-4 on page 5)

\begin{tabular}{|c|c|c|c|}
\hline 1 Plant name & 2 & 3 & 4 \\
\hline Acer negundo $\mathrm{L}$. & 4 & $\mathrm{O}$ & 5 \\
\hline Acer saccharinum L. & 1 & E & 4 \\
\hline Aesculus hippocastanum L. * & 2 & $\mathrm{O}$ & 7 \\
\hline Ailanthus altissima (Mill.) Swingle & 4 & $\mathrm{O}, \mathrm{S}$ & 4 \\
\hline Albizia julibrissin Durazz.** & 1 & & 8 \\
\hline Amelanchier canadensis Medik. & 1 & & 4 \\
\hline Amygdalus nana $\mathrm{L}$. & 1 & S & 4 \\
\hline Andrachne colchica Fisch. et Mey. & 1 & S & 5 \\
\hline Berberis julianae Schneid.* & 1 & E & 5 \\
\hline Berberis thunbergii DC. & 1 & & 4 \\
\hline Biota orientalis (L.) Endl.* & 2 & $\mathrm{E}$ & 5 \\
\hline Broussonetia papyrifera (L.) L'Hérit. in Vent.* & 1 & $\mathrm{E}$ & 7 \\
\hline Buddleja davidii Franch.* & 2 & $\mathrm{E}$ & 7 \\
\hline Campsis radicans (L.) Seemann & 1 & S & 4 \\
\hline Catalpa bignonioides Walter & 1 & $\mathrm{O}$ & 5 \\
\hline Celtis australis L.* & 1 & & 7 \\
\hline Celtis occidentalis $\mathrm{L}$. & 3 & $\mathrm{O}$ & 4 \\
\hline Cerasus $\times$ yedoensis (Matsum.) I. Tóth & 1 & & \\
\hline Cerasus serrulata (Lindl.) G. Don & 1 & & 5 \\
\hline Cercis siliquastrum L. ${ }^{*}$ & 2 & $\mathrm{O}$ & 7 \\
\hline Cercis siliquastrum L. 'Roseum'* & 1 & & 7 \\
\hline Clerodendrum bungei Steud.** & 2 & S & 8 \\
\hline Clerodendrum trichotomum Thunb.** & 1 & $\mathrm{~S}$ & 6 \\
\hline Cornus alba $\mathrm{L}$. & 2 & & 2 \\
\hline Cornus stolonifera Michx. & 2 & & 2 \\
\hline Corylus colurna L.* & 1 & $\mathrm{E}$ & 6 \\
\hline Cotoneaster acutifolius Turcz. & 2 & & 4 \\
\hline Cotoneaster bullatus Bioss & 3 & & 5 \\
\hline Cotoneaster dammeri Schneid.* & 2 & & 6 \\
\hline Cotoneaster dielsianus Pritz. & 3 & & 4 \\
\hline Cotoneaster divaricatus Rehd. ex Wils. & 3 & $\mathrm{O}$ & 4 \\
\hline Cotoneaster hebephyllus Diels & 3 & & 4 \\
\hline Cotoneaster horizontalis Dene. & 2 & $\mathrm{O}$ & 3 \\
\hline Cotoneaster insignis Pojark. & 3 & $\mathrm{O}$ & 4 \\
\hline Cotoneaster integerrimus Med. & 2 & & 6 \\
\hline Cotoneaster multiflorus Bunge & 3 & $\mathrm{O}$ & 6 \\
\hline Cotoneaster nebrodensis (Guss.) K. Koch. & 2 & & 6 \\
\hline Cotoneaster niger (Thunb.) Fries & 2 & & 6 \\
\hline Cotoneaster nitens Rehd. et Wils. & 3 & $\mathrm{O}$ & 5 \\
\hline Cotoneaster racemiflorus (Desf.) K. Koch & 3 & & 5 \\
\hline Cotoneaster salicifolius Franch. & 2 & & 6 \\
\hline Crataegus crus-galli L. & 2 & & 3 \\
\hline Crataegus flabellata (Bosc) K. Koch & 1 & & 3 \\
\hline Crataegus prunifolia (Poir.) Pres & 1 & & 3 \\
\hline Diospyros lotus L.** & 3 & & 8 \\
\hline Elaeagnus angustifolia $\mathrm{L}$. & 2 & $\mathrm{O}$ & 7 \\
\hline Euodia hupehensis Dode * & 2 & $\mathrm{E}$ & 7 \\
\hline Euodia velutina Rehd et Wils.* & 1 & & 7 \\
\hline Fraxinus pennsylvanica Marsh. & 3 & $\mathrm{O}$ & 4 \\
\hline Ginkgo biloba L.* & 1 & $\mathrm{~F}$ & 4 \\
\hline Gleditsia triacanthos L. & 1 & $\mathrm{E}$ & 3 \\
\hline Hedera hibernica (Kirchner) Bean * & 4 & $\mathrm{O}$ & 5 \\
\hline Hibiscus syriacus L.** & 2 & & 7 \\
\hline Ilex aquifolium L.* & 1 & & 5 \\
\hline Koelreuteria paniculata Laxm.* & 2 & $\mathrm{O}$ & 7 \\
\hline Laburnum alpinum (Mill.) Bercht. et Presl & 1 & & 6 \\
\hline Laburnum anagyroides Medik. & 2 & $\mathrm{E}$ & 6 \\
\hline Laurocerasus officinalis Roem.* & 3 & & 8 \\
\hline Lavandula angustifolia Mill.** & 1 & & 8 \\
\hline
\end{tabular}

\begin{tabular}{|c|c|c|c|}
\hline 1 Plant name & 2 & 3 & 4 \\
\hline Ligustrum amurense Carr. & 1 & & 3 \\
\hline Ligustrum delavayanum Hariot & 1 & & 4 \\
\hline Ligustrum ovalifolium Hasskarl * & 2 & & 6 \\
\hline Lonicera $\times$ amoena Zabel 'Alba' Zabel & 1 & & \\
\hline Lonicera × purpusii Rehd. & 2 & $\mathrm{~F}$ & \\
\hline Lonicera fragrantissima Lindl. et Paxt. & 1 & & 7 \\
\hline Lonicera japonica Thunb. 'Halliana'* & 1 & & 8 \\
\hline Lonicera korolkowii Stapf & 1 & & 4 \\
\hline Lonicera maackii (Rupr.) Maxim. & 1 & & 3 \\
\hline Lonicera morrowii A. Gray & 1 & & 3 \\
\hline Lonicera nitida Wils.** & 2 & & 7 \\
\hline Lonicera standishii Jacq. f. lancifolia Rehd. & 1 & & 7 \\
\hline Lonicera tatarica L. & 3 & $\mathrm{O}$ & 3 \\
\hline Lycium barbarum L. & 1 & $\mathrm{O}$ & 4 \\
\hline Mahonia aquifolium (Pursh) Nutt. & 3 & $\mathrm{O}$ & 4 \\
\hline Malus $\times$ purpurea (Barbier) Rehd. & 3 & & \\
\hline Malus $\times$ zumi (Matsum.) Rehd. & 1 & & \\
\hline Malus baccata (L.) Borkh. & 1 & & 3 \\
\hline Malus floribunda Van Houtte & 1 & & 4 \\
\hline Morus alba L.* & 3 & $\mathrm{O}$ & 6 \\
\hline Morus rubra L.* & 1 & & 6 \\
\hline Paeonia suffruticosa Andrews & 1 & & 5 \\
\hline Paliurus spina-christi Mill.* & 1 & & 7 \\
\hline Parrotia persica (DC.) C. A. Mey.* & 1 & & 7 \\
\hline Parthenocissus inserta (Kem.) Fritsch & 2 & $\mathrm{E}$ & 4 \\
\hline Parthenocissus quinquefolia (L.) Planch. & & & \\
\hline var. engelmannii Koehne et Graebn. & 1 & & 3 \\
\hline Parthenocissus tricuspidata (S. et Z.) Planch.* & 3 & $\mathrm{O}$ & 7 \\
\hline Paulownia tomentosa (Thunb) S. et Z. in St.** & 2 & $\mathrm{O}$ & 7 \\
\hline Platanus hybrida Brot. & 1 & $\mathrm{O}$ & 7 \\
\hline Populus $\times$ canadensis Mönch & 2 & $\mathrm{O}$ & \\
\hline Populus $\times$ canescens (Ait.) Sm. & 1 & & 4 \\
\hline Populus alba L. & 1 & & 4 \\
\hline Populus nigra L. & 1 & & 3 \\
\hline Populus nigra L. 'Italica' & 1 & & \\
\hline Prinsepia sinensis (Oliv.) Oliv. & 1 & & 4 \\
\hline Prunus cerasifera Ehrh. (P. divaricata Led.)* & 3 & $\mathrm{O}$ & 6 \\
\hline Ptelea trifoliata L. & 2 & $\mathrm{E}$ & 3 \\
\hline Pyracantha coccinea Roemer ** & 3 & $\mathrm{E}$ & 7 \\
\hline Pyracantha hybrids & 3 & & 7 \\
\hline Pyrus elaeagrifolia Pall. & 1 & & 4 \\
\hline Rhamnus utilis Dene. & 1 & & 5 \\
\hline Rhodotypos scandens (Thunb.) Makino & 1 & & 3 \\
\hline Robinia luxurians (Dieck) Schneid. & 2 & $\mathrm{~S}$ & 5 \\
\hline Robinia pseudoacacia L. & 2 & $\mathrm{O}$ & 4 \\
\hline Rosa nitida Willd. & 1 & & 3 \\
\hline Smilax excelsa L.** & 3 & & 8 \\
\hline Sophora japonica L.* & 2 & $\mathrm{O}$ & 7 \\
\hline Sorbus aria (L.) Cranitz & 2 & & 4 \\
\hline Spartium junceum L. ${ }^{* *}$ & 1 & & 8 \\
\hline Spiraea $\times$ schinabeckii Zabel in Wittm. & 1 & S & \\
\hline $\begin{array}{l}\text { Symphoricarpos rivularis Suksdorf } \\
\text { var. laevigatus (Fern.) Blake }\end{array}$ & 2 & & 3 \\
\hline Taxus baccata $\mathrm{L}$. & 2 & $\mathrm{O}$ & 6 \\
\hline Trachycarpus fortunei Wendl. ** & 1 & & 9 \\
\hline Toona sinensis (syn. Cedrela s. A. Juss.) Roem. & 3 & S & 6 \\
\hline Xanthoceras sorbifolium Bunge & 1 & $\mathrm{~F}$ & 6 \\
\hline Zanthoxylum simulans Hance.* & 1 & & 7 \\
\hline
\end{tabular}




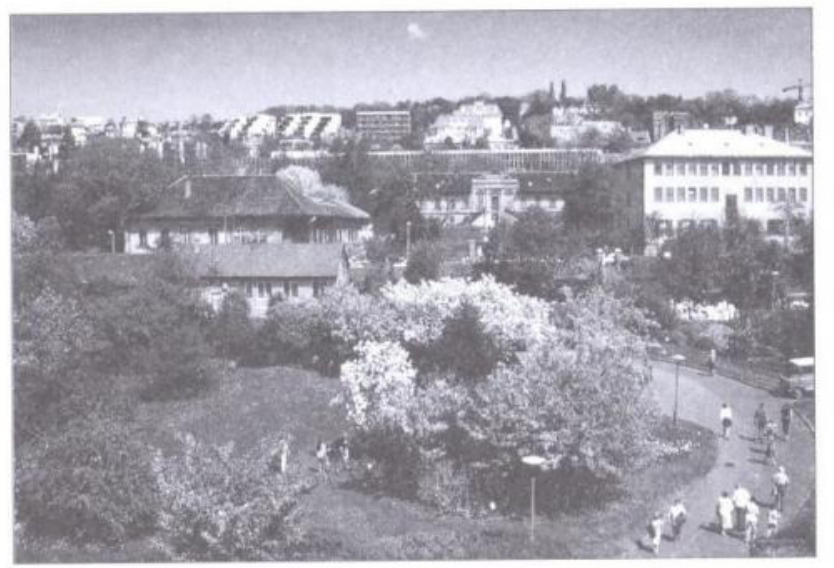

Figure $I$ General view of the Buda Arboretum in the spring on the southern slope of the Gellért Hill (background) and the sorrounding houses of the town Budapest. (In foreground: collection of flowering crabapples and Japanes cherries)

Figure 2 The Buda Arboretum in the autumn
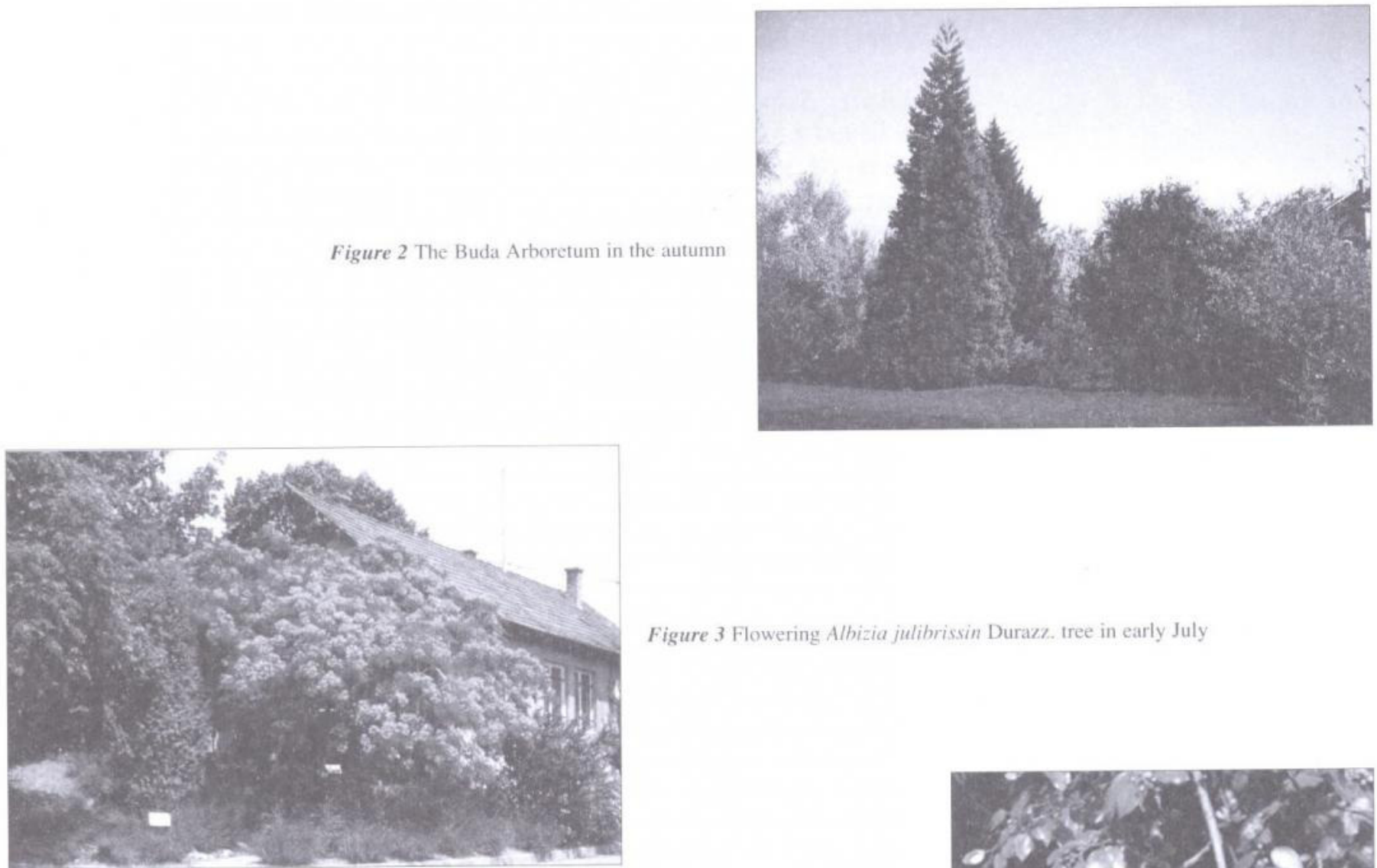

Figure 3 Flowering Albizia julibrissin Durazz. tree in early July

Figure 4 Paliurus spina-christi Mill.

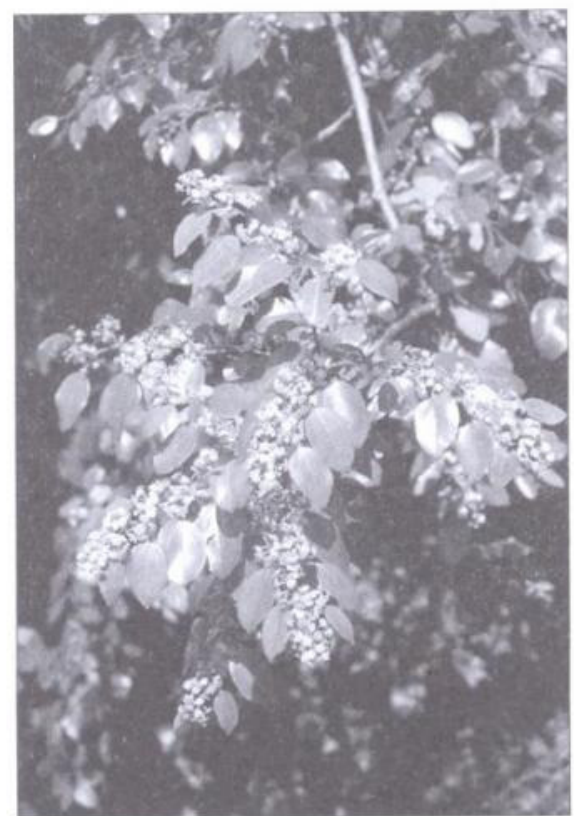




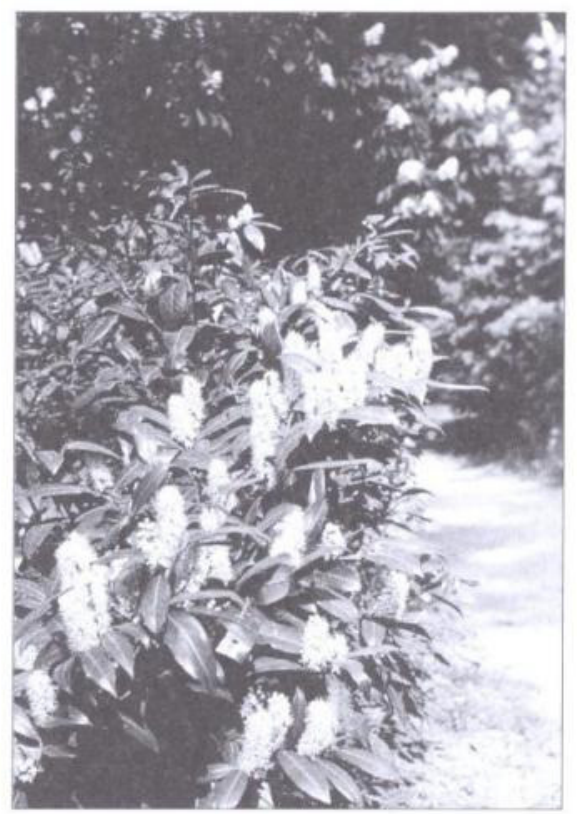

Figure 5 Laurocerasus officinalis Roem (syn. Prunus laurocerasus) varieties come almost true from seed, because they are generally representatives of geografical ecotypes

Figure 6 Smilax excelsa L. fruits in late October. This plant is behaving either as wintergreen or decidious, depending on the year.
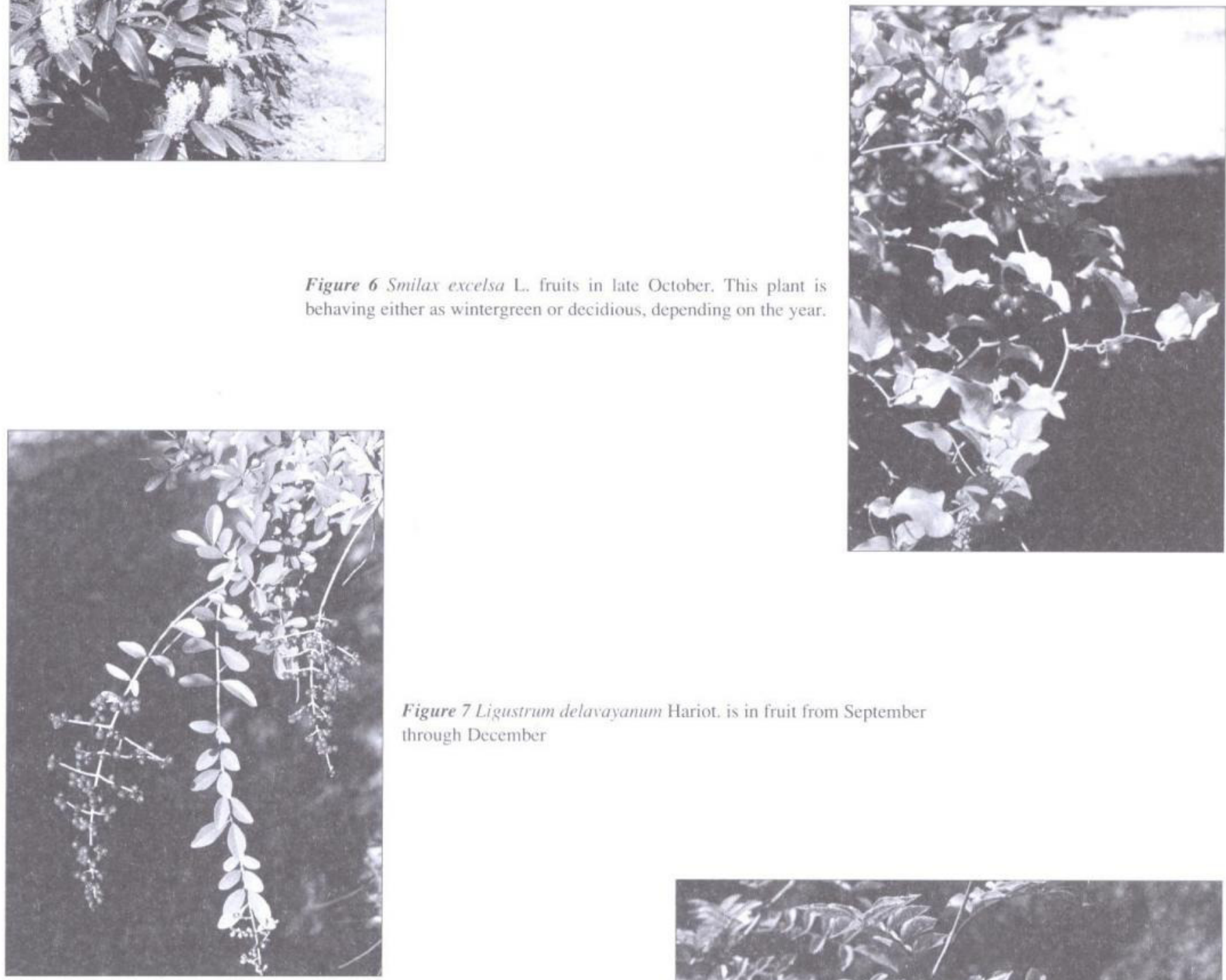

Figure 7 Ligustrum delavayanum Hariot, is in fruit from September through December

Figure 8 Zanthoxylum simulans Hance, fruited pricky branch. Seeds germinate freely, but most of them die in the first winter.

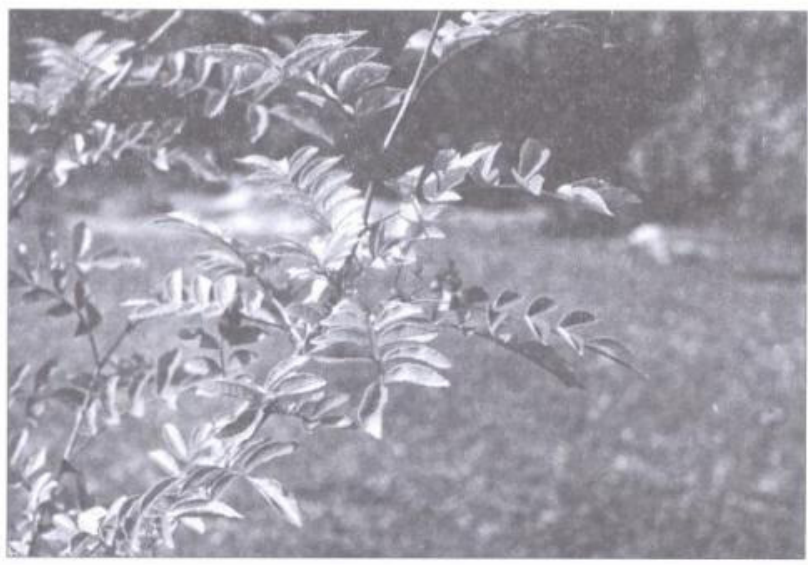


Legend:

Column 2: 4 - many seedlings all over the Arboretum

3 - many seedlings in some places

2 - few seedlings

$1-1-2$ seedlings per year

Column 3: $\mathrm{O}$-seedlings can be found outside the garden too

$\mathrm{E}$ - seedlings are common elsewhere outside the garden.

$\mathrm{S}$ - spreading by sprouts

F - 1-2 specimens for $6-8$ year

Column 4 : the numbers indicate the hardiness zone of the plant defined by Krüssmann (1986-1986).

One asterisk (*) or two (**) after the author's name in column 1 shows, that the northern border of the plant's natural distribution area is in zone $\mathbf{8}$ or $\mathbf{9}$. which is one or two zones more south than that of Hungary.

elements (from climatic zones 8 or 9 ) have the tendency to escape and almost naturalise in the Buda Arboretum. This clearly shows the greenhouse effect of the city of Budapest (Schmidt 1986) and the additional effect of the southern slope and the sheltered position in which the Arboretum is situated (Schmidt 1994).

The phenomenon of escaping from culture is common with many other non-native plants and was thoroughly studied and discussed from the botanical point of view by Priszter (1944, 1960, 1963, 1997) and Udvardy (1997. 1998a, 1998b, 1999b). The mentioned authors consider the "escaping" exotic plants as potential weeds and (therefore) dangerous to native vegetation.

Bartha (1994) and Bartha and Mátyás (1995) on the other hand made studies on the state of exotic trees in Hungarian arboreta taking them into account as potential reserves for afforestation and/or landscaping under extreme and urban conditions.

Most probably both sides have their right depending on the actual place of application or subspontaneous distribution of the given plants.

Anyway the results suggest a different approach to the urban plantings in Hungary with a stronger emphasise on heat-loving (and therefore heat-tolerant) woody plants. On the other hand the 110 species listed in table 1 can be also garden escapes in the future.

Following species have been planted, but do not spread in the Arboretum by themselves in spite of the observations of Udvardy (1997): Amygdalus communis L., Amorpha fruticosa L., Cydonia oblonga Mill em. Beck, Juglans nigra L., Padus serotina (Ehrh.) Borkh., Reynoutria aubertii (L.) Henry ex Hedberg Moldenke, Rhus hirta (L.) Sundw.

\section{References}

Bartha D. (1994): Magyarország faóriásai és famatuzsálemei. Erdészettörténeti Közlem. 15: 1-242.
Bartha D. \& Mátyás Cs. (1995): Erdei fa- és cserjefajok elôfordulása Magyarországon. - Sopron, 223.

Jackson, B. D. (ed.) et al. (1885-1991): Index Kewensis, et Suppl. 1-18. - Clarendon, Oxford

Krüssman, G. (1976-1978): Die Laubgehölze. (1-3). - Parey, Berlin-Stuttgart

Krüssman, G. (1984-1986): Manual of broad-leaved trees and shrubs. - Timber Press, Portland, OR

Priszter Sz. (1944): Adventív és szupspontán növények Budapestrốl. - Bot. Közlem. 41: 65-66.

Priszter Sz. (1960): Megjegyzések adventív növényekhez. 3. Néhány feltünốbb adventív-elốfordulás. - Bot. Közlem. 48: 272-277.

Priszter Sz. (1963): A magyar adventívflóra bibliográfiája. - Bot. Közlem. 50. 213-223.

Priszter Sz. (1997): A magyar adventívflóra kutatása. - Bot. Közlem. 84 (1-2): 25-32.

Schmidt G. (1986): Városfásítás (Urban Horticulture) in Schmidt G. (ed.) A kert élố díszei. - Mezôgazdasági Kiadó, Budapest

Schmidt, G. (1993): Magyar nemesítésû díszfák-díszcserjék és melegigényes exoták a Kertészeti és Élelmiszeripari Egyetem Budai Arborétumában. - A Kertészeti és Élelmiszeripaii Egyetem Közleményei Vol. LIII. Supl. pp. 56-61.

Schmidt G. (1994): The Buda Arboretum of the University of Horticulture and Food Industry. - Márton, Budapest, pp. 2-5, $21-46$.

Schmidt G. \& Keller Pintér J. (2000): A Kertészeti és Élelmiszeripari Egyetem Budai Arborétuma. - Interagent, Budapest. 3-6 (47 pp.)

Udvardy, L. (1997): Fás szárú adventív növények Budapesten és környékén. (Woody adventive plants in Budapest and in its surroundings.) - Kandidátusi értekezés. KÉE Növénytani Tanszék és Soroksári Botanikus Kert, Budapest. 126.

Udvardy L. (1998a): Spreading and coenological circumstances of the tree of heaven (Ailanthus altissima) in Hungary. - Acta Bot. Hung. 41: 299-314.

Udvardy L (1998b): Classification of adventives dangerous to the hungarian natural flora (Acta Botanica Hungarica 41 (1-4): 315-331.

Udvardy L. (1999a): Gap-inhabitant woody alien plants in Budapest. Publ. Univ. Hort. Ind. Alim, 59: 175-176.

Udvardy L. (1999b): Exotic woody plants inclining to escape in an arboretum under strong urban effect in Budapest. Publ. Univ. Hort. Ind. Alim, 59: 171-174.

Udvardy L. \& Facsar G. (1997): Arboreta and living plant collections as local naturalization centres of phanerophyta in Budapest; in: Pavel Eliáš (ed.) Invázie a invázne organizmy. Príspevky z Vedeckej Konferencie Nitra, 19-20. November 1996. - Nitra, pp. 70-74.

Wittig, R. (1991): Ökologie der Großstadtflora. - Gustav Fischer, Stuttgart. 